\title{
Conjectures on the Taylor Series Expansion Coefficients of the Jacobian Elliptic Function $\operatorname{sn}(x, k)$
}

\section{By Arne Fransén}

\begin{abstract}
Two conjectures are posed for the coefficients introduced by Alois Schett in the Taylor series expansion of the Jacobian elliptic function $\operatorname{sn}(x, k)$. The first conjecture is furnished with a proof revealing a procedure which might be useful when calculating further coefficients. Some of the coefficients are tabulated.
\end{abstract}

The Taylor series expansion coefficients of the Jacobian elliptic function $\operatorname{sn}(x, k)$ were given by Schett [2], [3] for powers of $x$ up to and including 49. In accordance with Wrigge [7] we write

$$
\operatorname{sn}(x, k)=\sum_{n=0}^{\infty}(-1)^{n} \frac{x^{2 n+1}}{(2 n+1) !} s_{2 n+1}(1, k),
$$

where we have the polynomials

$$
s_{2 n+1}(1, k)=\sum_{j=0}^{n} c_{j}^{(2 n+1)} k^{2 j}
$$

The coefficients $c_{j}^{(2 n+1)}$ may be obtained using some rather complicated recurrence formulae or in a combinatorial way as in Schett [4] and in Dumont [1], where they are denoted by $a_{2 n+1,0, j}$.

CONJECTURE I. The coefficient $c_{j}^{(m)}$ of $k^{2 j}$ in the polynomials $s_{m}(1, k)$, where $m=2 n+1$, is obtained as a solution to the difference equation over $m$, the characteristic equation of which is

$$
\prod_{p=0}^{j}(u-[2(j-p)+1])^{p+1}=0
$$

Proof. Jacobi in "Fundamenta Nova Theoriae Functionum Ellipticarum," 1829, and afterwards Glaisher [Messenger of Math., Vol. XI, 1882], [5, p. 516] gives the formula

$$
\begin{aligned}
\frac{d^{2} \operatorname{sn}^{n}(x, k)}{d x^{2}}= & n(n-1) \operatorname{sn}^{n-2}(x, k)-n^{2}\left(1+k^{2}\right) \operatorname{sn}^{n}(x, k) \\
& +n(n+1) k^{2} \operatorname{sn}^{n+2}(x, k) .
\end{aligned}
$$

Putting $n=1$, we get

$$
\frac{d^{2} \operatorname{sn}(x, k)}{d x^{2}}=-\left(1+k^{2}\right) \operatorname{sn}(x, k)+2 k^{2} \operatorname{sn}^{3}(x, k) .
$$

Received July 7, 1980; revised December 5, 1980.

1980 Mathematics Subject Classification. Primary 33A25.

$K e y$ words and phrases. Elliptic functions. 
Now, by writing

$$
\operatorname{sn}(x, k)=\sum_{n=0}^{\infty}(-1)^{n} r_{2 n}(x) k^{2 n}
$$

and differentiating twice with respect to $x$, we get for $n \geqslant 1$ the differential equations

$$
\begin{aligned}
r_{0}^{\prime \prime}+r_{0} & =0, \\
r_{2 n}^{\prime \prime}+r_{2 n} & =r_{2 n-2}-2 \sum_{j=0}^{n-1} r_{2 j} \sum_{i=0}^{n-1-j} r_{2 i} r_{2 n-2 j-2 i-2},
\end{aligned}
$$

with the obvious initial conditions

$$
\begin{gathered}
r_{0}(0)=r_{2 n}(0)=0, \\
r_{0}^{\prime}(0)=1, \quad \text { and } \quad r_{2 n}^{\prime}(0)=0 .
\end{gathered}
$$

These differential equations may be easily solved recursively, using de Moivre's theorem to linearize in the right member. The calculations are quite lengthy and therefore will be omitted, but there is no difficulty in proving by induction that

$$
r_{2 n}=\sum_{j=0}^{n} \sum_{i=0}^{n-j} b_{j i}^{(n)} x^{i}\left\{\begin{array}{c}
\sin \\
\cos
\end{array}\right\}(2 j+1) x,
$$

where the sine is chosen for even values of $i$ and the cosine for odd values of $i$ and where the coefficients $b_{j i}^{(n)}$ are real. From (6) we see that $r_{2 n}$ are odd functions of $x$.

The result follows by introducing the Taylor series expansion for the sines and cosines and identifying coefficients of like powers of $k$ in (1) and (2) as was done by Wrigge [7] for $n=1,2$.

Example 1. The comprehensive Tables I-X show calculated values of the coefficients $c_{j}^{(m)}$ for $j=1$ to 10 and $m<100$. Using the procedure described in the proof, we may easily extend these tables to values of $j>10$. Note the periodic behavior of the last digit in the Tables I-X.

Formulae for the coefficients $c_{j}^{(m)}$ for $j<10$ are presented in Table XI. These formulae may be used to extend the Tables I-X to values of $m>100$.

The values and expressions in Tables I-XI have been verified directly and found to be in agreement with those published by Schett [2], [3]. Conjecture I has also been proved by Wrigge [7] for $j \leqslant 2$.

The special structure of the coefficients given in Table XI leads to

CONJECTURE II. The coefficient $c_{j}^{(m)}$ of $k^{2 j}$ in the polynomials $s_{m}(1, k)$, where $m=2 n+1, i s$

$$
4^{-2 j} \sum_{p=0}^{j} \frac{(-1)^{p}}{p !} P_{p}(n ; j)[2(j-p)+1]^{m},
$$

where $P_{p}$ are polynomials of degree $p$ with integral coefficients defined by

$$
P_{p}(n ; j)=\left(\sum_{i=0}^{p}(-1)^{[i / 2]} A_{i}(j, p) D^{i}\right) \beta^{p},
$$

where $D$ is the differentiation operator with respect to $\beta$ and

$$
\beta=4[2(n-j)+3]=4[m-2 j+2] \text {. }
$$


We have further for $i \geqslant 1$

$$
\begin{gathered}
A_{0}(j, p) \equiv 1, \\
A_{i}(j, p)=\sum_{n=0}^{[i / 2]} Q_{i-2 n}^{(i)}(p) j^{n}+\sum_{n=0}^{[(i-1) / 2]} Q_{i-2 n-1}^{(i)}(p) \delta_{j, p+n},
\end{gathered}
$$

where $Q_{r}^{(i)}$ are polynomials of degree $r$ and $\delta_{j, p+n}$ is the Kronecker symbol. (The brackets indicate, as usual, the integer part.)

Some of the polynomials $Q_{r}^{(i)}$ have been empirically determined. They are all numbered below to make it easier to refer to a possible wrong formula.

We have the following expressions

(17) $Q_{0}^{(1)}(p)=-1$,

(18) $Q_{1}^{(1)}(p)=p-1$,

(19) $Q_{0}^{(2)}(p)=4$,

(20) $Q_{1}^{(2)}(p)=p-3$,

(21) $Q_{2}^{(2)}(p)=-\left(3 p^{2}-7 p-106\right) / 6$,

(22) $Q_{0}^{(3)}(p)=1$,

(23) $Q_{1}^{(3)}(p)=4(p-3)$,

(24) $Q_{2}^{(3)}(p)=\left(3 p^{2}-43 p-72\right) / 6$,

(25) $Q_{3}^{(3)}(p)=-(p-3)\left(p^{2}-p-114\right) / 6$,

(26) $Q_{0}^{(4)}(p)=8$,

(27) $Q_{1}^{(4)}(p)=-(p-4)$,

(28) $\quad Q_{2}^{(4)}(p)=-\left(6 p^{2}-38 p-166\right) / 3$,

(29) $Q_{3}^{(4)}(p)=-\left(p^{3}-34 p^{2}+45 p+498\right) / 6$,

(30) $Q_{4}^{(4)}(p)=\left(15 p^{4}-90 p^{3}-3475 p^{2}+23022 p+18352\right) / 360$,

(31) $Q_{0}^{(5)}(p)=-1$,

(32) $Q_{1}^{(5)}(p)=8(p-5)$,

(33) $Q_{2}^{(5)}(p)=-\left(3 p^{2}-49 p-82\right) / 6$,

(34) $\quad Q_{3}^{(5)}(p)=-2(p-5)\left(p^{2}-5 p-111\right) / 3$,

(35) $\quad Q_{4}^{(5)}(p)=-\left(15 p^{4}-930 p^{3}+7985 p^{2}+38762 p-41760\right) / 360$,

(36) $\quad Q_{5}^{(5)}(p)=(p-5)\left(3 p^{4}-10 p^{3}-1255 p^{2}+6542 p+51120\right) / 360$.

In particular, we have

(37) $P_{0}(n ; j) \equiv 1$,

(38) $\quad P_{1}(n ; j)=\beta-\delta_{j 1}$,

(39) $\quad P_{2}(n ; j)=\beta^{2}+2\left(1-\delta_{j 2}\right) \beta-2\left(4 j+18-\delta_{j 2}\right)$,

(40) $\quad P_{3}(n ; j)=\beta^{3}+3\left(2-\delta_{j 3}\right) \beta^{2}-4(6 j+25) \beta-6\left(\delta_{j 4}-29 \delta_{j 3}\right)$,

(41) $\quad P_{4}(n ; j)=\beta^{4}+4\left(3-\delta_{j 4}\right) \beta^{3}-4\left(12 j+43+3 \delta_{j 4}\right) \beta^{2}$ $-8\left(12 j+51+3 \delta_{j 5}-98 \delta_{j 4}\right) \beta+24\left(8 j^{2}+74 j+147-33 \delta_{j 4}\right)$,

$$
\begin{aligned}
P_{5}(n ; j)= & \beta^{5}+5\left(4-\delta_{j 5}\right) \beta^{4}-20\left(4 j+11+2 \delta_{j 5}\right) \beta^{3} \\
& -20\left(24 j+94+3 \delta_{j 6}-106 \delta_{j 5}\right) \beta^{2} \\
& +8\left(120 j^{2}+1030 j+1863-15 \delta_{j 6}+5 \delta_{j 5}\right) \beta \\
& -120\left(\delta_{j 7}-42 \delta_{j 6}+680 \delta_{j 5}\right) . \square
\end{aligned}
$$

Example 2. The polynomials $P_{p}(n ; j)$ corresponding to the expressions given in Example 1 and in Table XI are listed in Table XII. 
The conjectured expressions in Eqs. (17)-(42) seem to be generally valid. Eqs. (37)-(42) are only consequences of Eqs. (17)-(36). The expressions in Conjecture II have been verified directly and found to be in agreement with the results from Conjecture I.

Perhaps Conjecture II may be proved in a similar way as Conjecture I, or, alternatively, by use of the technique in [7], Theorem VIII in [6], the recurrence formulae (2) in [1], or Corollary 1 in [1]. However, there will be a great amount of labor doing so. I look forward to more elegant proofs which hopefully might lead to an explicit expression for the polynomials $P_{p}(n ; j)$. Thus we need only a recurrence formula for the polynomials $Q_{r}^{(i)}(p)$, which is probably an impossible task to perform.

Similar conjectures may be posed for the corresponding coefficients in the Taylor series expansions of the Jacobian elliptic functions $\operatorname{cn}(x, k)$ and $\operatorname{dn}(x, k)$.

The necessary computer calculations were carried out using REDUCE and the FORTRAN subroutine package NUMBIG for handling large numbers.

Acknowledgements. I am greatly indebted to my colleague Dr. Staffan Wrigge who made me interested in this particular problem and presented a number of appropriate references. Also I would like to thank the unknown referee for many valuable suggestions.

National Defence Research Institute

Division 1, Section 185

S-104 50 Stockholm 80, Sweden

1. D. Dumont, "A combinatorial interpretation for the Schett recurrence on the Jacobian elliptic functions," Math. Comp., v. 33, 1979, pp. 1293-1297.

2. A. SChetT, "Properties of the Taylor series expansion coefficients of the Jacobian elliptic functions," Math. Comp., v. 30, 1976, pp. 143-147.

3. A. SCHETT, Addendum to "Properties of the Taylor series expansion coefficients of the Jacobian elliptic functions," Math. Comp., v. 31, 1977, Microfiche supplement.

4. A. SCHETr, "Recurrence formula of the Taylor series expansion coefficients of the Jacobian elliptic functions," Math. Comp., v. 31, 1977, pp. 1003-1005.

5. E. T. Whitraker \& G. N. Watson, A Course of Modern Analysis, 4th ed., Cambridge Univ. Press, New York, 1962.

6. S. WrigGe, "Calculation of the Taylor series expansion coefficients of the Jacobian elliptic function $\operatorname{sn}(x, k)$," Math. Comp., v. 36, 1981, pp. 555-564.

7. S. WRIGGe, "A note on the Taylor series expansion coefficients of the Jacobian elliptic function $\operatorname{sn}(x, k)$," Math. Comp., v. 37, 1981, pp. 495-497. 
TABle I. Coefficients $c^{(m)}$ for $m<100$

cofficient 
TABLE II. Coefficients $c_{2}^{(m)}$ for $m<100$

m

1 coefficient

\section{0}

116294673

2949965020

74197080276

185539731885

46535238000235

1163848723925346

29100851707716150

727566807977891803

18189614152200873621

454744658216502193656

11368657974646161302248 284216848055029040209305

7105425014717554019615631

177635661714292879129333150

4440891888211006424569211370

111022300477586804328521775591

2775557542867631254917084671065 69388938863336838872742230963028

1734723474327885153932200566560316

43368086883974153468865596179537861 1084202172341038681140525152576628723 27105054310788355233787656136744958490 677626357790855396141703190125407409886 169406589444968768734261555200748618699315 423516473626059259570422483352507810221405 10587911840668614140822229932564269315050736 264697796016874697910341197721597886185564880 6617444900423347901998172936625910415385519153 165436122510597438830695275898188315660019925911 4135903062765063397027307959659974862336914826966 103397576569127765554621350960108392056172432974098 2584939414228205068659375498399045545726053194512575 6462348535570522782182507689652851380624977979163041 1615587133892631630128648676699398918500088983590130380 40389678347315799386175659899126556642487220706327730340 1009741588682895064346445681406608133949168820493538050781 25243548967072377343848402464364230992616225277932125097595 631088724176809440374524360436484290756525665199326327860178 15777218104420236071822598295039799316698454842488488223670406 394430452610505902370792306285275779986366286193975266846360971 9860761315262647564564681305484605791028835257008185041541038373 246519032881566189162831343045812203457793789138390278743779171560 6162975822039154729518756397985282528121213141022235644288093575096 
TABLE III. Coefficients $c_{3}^{(m)}$ for $m<100$

\section{TABLE III. COefficients $c_{3}$ for $m<100$}

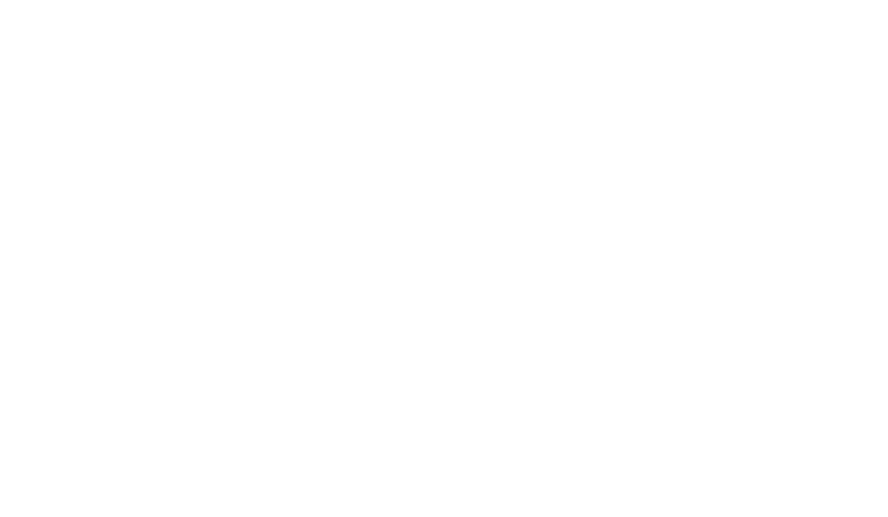

$$
\begin{aligned}
& 0 \\
& 0 \\
& 0 \\
& 1228 \\
& 165826 \\
& 13180268 \\
& 834687179 \\
& 47152124264 \\
& 2504055894564 \\
& 128453495887560 \\
& 6460701405171285 \\
& 321298267540551700 \\
& 15875718186751193446 \\
& 781562415106660985428 \\
& 38396599486084770569951 \\
& 188415272554433297404688 \\
& 92396925087242863212482504 \\
& \text { 4529421792220618780953132624 } \\
& 221994390052130259394532925609 \\
& 1087912843407564265164178595980 \\
& 533114507941647087221696108146570 \\
& 26123594546702044085526699031503100 \\
& 1280082056495642083638458387900885491 \\
& 62724702167664030806105463320712967928 \\
& 150603352927474237430404905305182067706008 \\
& 361599566789909704272324537843571128856976484 \\
& 868201180758052308058092498645580622348221305700 \\
& 2084551453146368331328407816966461069963353999847200 \\
& 5005008319149989755864557792931267995261245675448141728 \\
& 12017025161122682167292682866887544425611761756718064677228 \\
& 1413791000362820909069430781859101554523460598462523339446959899 \\
& 3394512193982705386673372304598880348082747847203332386248895177140 \\
& 8150223779143940442146143569148734403748289709497311935946014637417381
\end{aligned}
$$$$
3073528273867302640364620157246357015276
$$$$
7379576501562696222059774285180327454181629
$$$$
17718387053299667526031545607505598124208043054
$$$$
42541863439091685468680525191321502602258565710855
$$$$
102143024946998883912060618070412257387835508479989136
$$$$
245245410136434447947233510301682741729736833122062014801
$$$$
\begin{array}{r}
58883423455557650589751950727047727706594157791796053891554 \\
28852877535978146700312837959228814345064795180987018772648844
\end{array}
$$$$
69275759046050999387523149899608772486006904428452001460999318920
$$$$
166331097523792400504281484743484618065720631566238058487324251377000
$$$$
399360965190308824688674674124147749478467527758766757429904973600699828
$$
1 1568687294639099050023021729003768183122137142701256787941737537946081526 \$88865677445354346075330175002928043890960370572479662512477698603070911348 4698441815028058438727557403259497589083457219075019327136964660397663743279 23022364915615355796559342935235689566050015238241056954724432903440109251142704 2302236491561535579659342935235689566050015238241056954724432903440109251142704
11280958808665461535744698182739734225534785161397399261557122602565598173389144 


\section{TABLE IV. Coefficients $c_{4}^{(m)}$ for $m<100$}

m

7

9

13

15

19 cefficient 0 458814920174904775826257436 37524907781760654616571819884 3058692313447287528959880082164 2487664722866600818433970414904068 $2020325386895 \$ 18771392559825506285$ 1639243235717722648852313037046453305 132923444218451509189072119405846654355 10774309557783598613466933578712446737055 873107580262755356033649662814680367103746 7074175457601087130862469171525732615146714 5731113290279152541326385472093837037802652646 464273113615330874205458871170525051939811187614 37608834197862575635540194937171017460468024168059 3046454243478505849181714021377418216044082870191023 246769872217866505286387899911965804995809092599313805 19988720387250704386816630085561606423919839750356146145 1619104708131727658393199917087006933289769137469787558520 131148414199504505950511524085219752842782306573102992452120 1062306889345217592537188378523502804346389351687829600463656 860470980271121894987621790622004366806344536467680361737790024 69698270921061772033660484086640334795499619278622518952808851481 5645566091314378121385665969345392830216205191730285628055448714869 457291164006523479765911360005829662217088143745179587927784380053079 37040599966067345943430184518726212084974014280130860729196966092967603 3000289388267519811041906205768838832567190131414282613433153417262036350 243023480317868437982345960763515177049403956655712430259455436590047907110 1968490391360130627756000727754390934946489175178004339548723280609496814010 1594477318047847555770749910058986247584147261867723635803045251560220136862210 1291526678435401783553160535531790668442356714153324310863614078628407143131591 10461366350718091687075961902785517739932518942731607165257860318695580126342603659 84737068723543994831773327645527906587133 क77809280686414100446520669875276621803544 68637026309948938219042131784988507624527449867680275757198120676999223732320791536149 555959916340765150484383038845850403034722698448234286657035712309321191785277562820724 45032753385564314370647382829435122695590797150942787207082105644630092709645293824610308 
TABle V. Coefficients $c_{\xi}^{(m)}$ for $m<100$

\section{TABLE V. Coefficients cf for $m<100$}
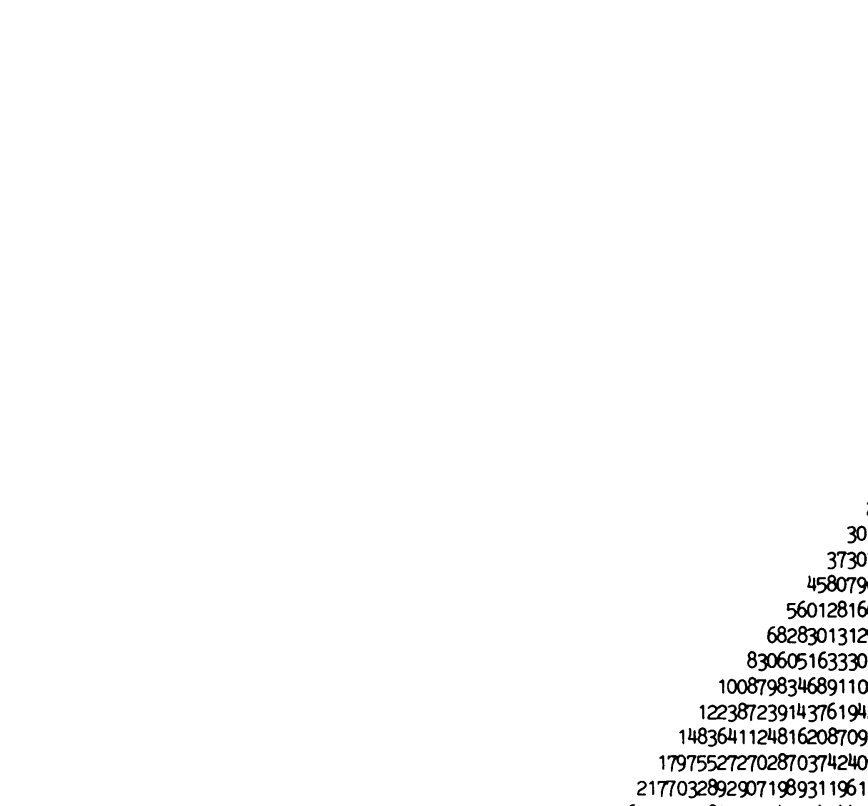

0

0

0

1

99642

116294673

47152124264

11966116940238

2347836365864484

393938089395885894

59752013018382750024

8470841585571575617239

1146456994425541774291534

150221961163114696686151695

19239380962379456298762250416

2424371762015227695363084225932

301977301501927982712251650296648

37303324488483426954302995423715292

4580796878616173620118408244176914608

560128160549135541529462577248201125213

68283013125971106451240903324125753716898

8306051633303508006760284701965046518394653

1008798346891106069674845062004217099062126168

122387239143761942370134589735761526561609251514

1483641124816208709358930724630959446874364584940

1797552727028703742409838111858884788669326444960066

217703289290719893119610564691182352540852373464053432

2635900338925094137454436781749489920440891125650500371

3190869731746670456494579893507932023000521229249637555382

38621603199608521151466631639698533360911531112660503019707

46742324156362328453362456356889930862379745555497650995931872

5656678540537174782458944367931781198896985172716772125630795992

684530169546542728974860447157249316167333115274473396060302763152

82834200357474696225136733741844478645992684053740898922870062933208 10023445491138254021182251750947543065340812214333320206188809840618464 1212879385536427560757054321273270313613301699778251975024971065577768825 146761 क5 34416982520417689677633682655914041238410388100972995955762393866 177584941143091540259210919896555905394744137836837915237623638531140613225 2148802586514895133475760425426106930300995075825118454047542594577823629498056 260007181667927050037249417014472081102294141321935465167822720380491496091361126 31461041406983193820596042698912468917715834478657102613130971418104631603437046196 3806800370382616050708887360179398042144644597948480420894664127065652370494315515006 460624039877059039364345942355441035601240515541927686344786090463447209790789079021736 573560820810269961426464467521428585781609721004572922693508377914333153099353793280527 6744016852429194007103161314193977682602690568114933471146495086918078488727901123433566238 816026725090505511883465047593210372947771351318924327404354903174522013771013100970746433959 9873929067036457934628925794574643712321155216213857203865378272839638771432232746250370157200 11947458893993607944703746710663034978283956573688437242242559689242698633062536818609092374021604 
TABLE VI. Coefficients $c_{6}^{(m)}$ for $m<100$ 59783653857084068659552416713877318073658259206485137263443032246658120866637083 10104800470571704821773137144065716653614478225968370497991628133153823537248631660 17078813576205170044222924934136890198577657408166641029617322498429159439484133324436 2386531530551672154178089647431424714548706489734247397865577694391054459033193854999514 487850240234559884235982684251132764026368160362486568251919172922303911999161896248886430 82449977774072786731056686992049978367005256294370333489760246369097792146183262782604429868 13934455047850968511717613860711038124028193567211341013786388753685261751869834349371300296244 2354973706110503837565603249686337152881887406205964978562269449759748504067920582702519698172963 397996865361870931299727260335792973157928488403482416699536274433298544294234439526267359939385721 67262253223920482917658922105017745926465509151636274380933733317077570675046600176409618503084776216 $11367417904945257138758662685765408094 \$ 15806260743082281257923951693161364296999805232832466666186499176$ 
TABLE VII. Coefficients $c_{7}^{(m)}$ for $m<100$ $5 \% 550040859679089778481316157348108115791055527611804495573003691354131170977392$ 13437946383534301678105789137377791472866606290333516744584792336522884435103414815 3026457753315643384723719397594441662693792859803550202426244392499022771906720960393 681463267084845722470307375279311919328562008684459536562621584490305778405608462991480 15341831136517909718765600526758453911 553807900766012145951479705863350986866314565102340 34534653553038507472258882447456568035772737193734488215103118770602798700652546592010735656 7773003213075635012355961194840929382429051158913581890201716691405762760070900301960120529890 1749396747506108758200898576082698740052699853721355310084514755156179859094027357793940550661928 39369618290024073135867239901835177841473096414668552941964017751017499278364286471123581497192116 88595875282335566617040573834587895798073815199208236810323518771550681940781475280174915823674759096 19936543444119121778220366273615733721445974023943846233343178494410197345253371456544097246937171586939 448615112915850086269446822165641867148034545197632361046754817772016940762897867130262547743445642702895 1009458362654244304897308185990562848826484147610289686243443167875482369814521517027000081375172948540901512 
TABLE VIII. Coefficients $c_{8}^{(m)}$ for $m<100$ 150895976111580246356909001162888264027760218873479742837062672337239599059305076 43829125679335574630014061598741241705681559040846683402506806117988329717912897108 12718143344655483038960398902652372104963548878706419075147444211203629858658568709444 3687580399440309646452396939916563651788852920538027068872359679905044099193394675075300 10685183686421769724102520494814463054672586443681877587235088493102869014413610795924907245 309455779372809954525810807422583651413455463671352077961638314215420908240253881628745922245 8958485368205197375078157564113938824998249695033814526713672407582209367379121657459157412585 25925372371968293109315257995161364187544758306258873084996217708226887115091155776423760830224241 7500637636043273733423617159224562849183066230261071976477174317849387389981368577434798322407085683 2169586855785888483164412771637468998478739494070751150310047101376068987892205438596119320641602505531 627451363325143887208259403623391293745650383661166822121788605115905531076817728621855381324459972211663 181435465792330460742305067561772920368669590169914589288166794997115711144003686619871388697384801145178919 52458445135265793453017642441049708151847889168227518761926510780204460499582031992548139076063604494164148610 1516594367131594496331164251523862269101150984305054849839348998217990222683196775663914196803569082893444881266 
TABLE IX. Coefficients $c_{9}^{(m)}$ for $m<100$ 
ARNE FRANSÉN

TABLE X. Coefficients $c_{10}^{(m)}$ for $m<100$

cefficient 
TABLE XI. Some expressions for the coefficients $c_{j}^{(m)}$

$$
\begin{aligned}
& 4^{0} c_{0}^{(m)}=1 \\
& 4^{2} c_{1}^{(m)}=3^{m}-(4 m-1) \\
& 4^{4} c_{2}^{(m)}=5^{m}-(4 m-8) 3^{m}+\left(8 m^{2}-32 m+7\right) \\
& 4^{6} c_{3}^{(m)}=7^{m}-(4 m-16) 5^{m}+\left(8 m^{2}-60 m+82\right) 3^{m}- \\
& \left(\frac{32}{3} m^{3}-120 m^{2}+\frac{1000}{3} m-67\right) \\
& 4^{8} c_{4}^{(m)}=9^{m}-(4 m-24) 7^{m}+\left(8 m^{2}-92 m+230\right) 5^{m}- \\
& \left(\frac{32}{3} m^{3}-176 m^{2}+\frac{2488}{3} m-945\right) 3^{m}+ \\
& \left(\frac{32}{3} m^{4}-\frac{704}{3} m^{3}+\frac{5008}{3} m^{2}-\frac{11716}{3} m+738\right) \\
& 4^{10} c_{5}^{(m)}=11^{m}-(4 m-32) 9^{m}+\left(8 m^{2}-124 m+442\right) 7^{m}- \\
& \left(\frac{32}{3} m^{3}-240 m^{2}+\frac{4936}{3} m-3264\right) 5^{m}+ \\
& \left(\frac{32}{3} m^{4}-\frac{928}{3} m^{3}+\frac{9160}{3} m^{2}-\frac{34376}{3} m+11661\right) 3^{m}- \\
& \left(\frac{128}{15} m^{5}-\frac{928}{3} m^{4}+\frac{12256}{3} m^{3}-\frac{69728}{3} m^{2}+\frac{733832}{15} m-8808\right) \\
& 4^{12} c_{6}^{(m)}=13^{m}-(4 m-40) 11^{m}+\left(8 m^{2}-156 m+718\right) 9^{m}- \\
& \left(\frac{32}{3} m^{3}-304 m^{2}+\frac{8152}{3} m-7440\right) 7^{m}+ \\
& \left(\frac{32}{3} m^{4}-\frac{1184}{3} m^{3}+\frac{15400}{3} m^{2}-\frac{81292}{3} m+46519\right) 5^{m}- \\
& \left(\frac{128}{15} m^{5}-384 m^{4}+\frac{19360}{3} m^{3}-49176 m^{2}+\frac{2402192}{15} m-150486\right) 3^{m}+ \\
& \left(\frac{256}{45} m^{6}-\frac{1536}{5} m^{5}+\frac{58208}{9} m^{4}-\frac{198304}{3} m^{3}+\frac{14730424}{45} m^{2}-\frac{9599332}{15} m+\right. \\
& \text { 110728) } \\
& 4^{14} c_{7}^{(m)}=15^{m}-(4 m-48) 13^{m}+\left(8 m^{2}-188 m+1058\right) 11^{m}- \\
& \left(\frac{32}{3} m^{3}-368 m^{2}+\frac{12136}{3} m-13984\right) 9^{m}+ \\
& \left(\frac{32}{3} m^{4}-480 m^{3}+\frac{23176}{3} m^{2}-51956 m+120337\right) 7^{m}- \\
& \left(\frac{128}{15} m^{5}-\frac{1408}{3} m^{4}+9824 m^{3}-\frac{288944}{3} m^{2}+\frac{6463012}{15} m-668241\right) 5^{m}+ \\
& \left(\frac{256}{45} m^{6}-\frac{5504}{15} m^{5}+\frac{84224}{9} m^{4}-\frac{356320}{3} m^{3}+\frac{34587544}{45} m^{2}-\frac{33993476}{15} m+\right. \\
& \text { 2004451) } 3^{\mathrm{m}}- \\
& \left(\frac{1024}{315} m^{7}-\frac{11008}{45} m^{6}+\frac{337408}{45} m^{5}-\frac{1074656}{9} m^{4}+\frac{46720768}{45} m^{3}-\right. \\
& \left.\frac{209978872}{45} m^{2}+\frac{906028148}{105} m-1443574\right)
\end{aligned}
$$


TABLE XI. Some expressions for the coefficients $c_{j}^{(m)}$ (continued)

$$
\begin{aligned}
4^{16} c_{8}^{(m)}= & 17^{m}-(4 m-56) 15^{m}+\left(8 m^{2}-220 m+1462\right) 13^{m}- \\
& \left(\frac{32}{3} m^{3}-432 m^{2}+\frac{16888}{3} m-23408\right) 11^{m}+ \\
& \left(\frac{32}{3} m^{4}-\frac{1696}{3} m^{3}+\frac{32488}{3} m^{2}-\frac{264236}{3} m+253307\right) 9^{m}- \\
& \left(\frac{128}{15} m^{5}-\frac{1664}{3} m^{4}+\frac{41632}{3} m^{3}-\frac{496432}{3} m^{2}+\frac{13907612}{15} m-1907640\right) 7^{m}+ \\
& \left(\frac{256}{45} m^{6}-\frac{2176}{5} m^{5}+\frac{119936}{9} m^{4}-\frac{620864}{3} m^{3}+\frac{76174744}{45} m^{2}-\right. \\
& \left.\frac{101048912}{15} m+9677802\right) 5^{m}- \\
& \left(\frac{1024}{315} m^{7}-\frac{2560}{9} m^{6}+\frac{460288}{45} m^{5}-\frac{1744480}{9} m^{4}+\frac{92504128}{45} m^{3}-\right. \\
& \left.\frac{106684168}{9} m^{2}+\frac{3405419728}{105} m-27334691\right) 3^{m}+ \\
& \left(\frac{512}{315} m^{8}-\frac{10240}{63} m^{7}+\frac{20480}{3} m^{6}-\frac{7004288}{45} m^{5}+\frac{31106848}{15} m^{4}-\right. \\
& \left.\frac{144814528}{9} m^{3}+\frac{4235963624}{63} m^{2}-\frac{12493128428}{105} m+19333223\right)
\end{aligned}
$$$$
4^{18} c_{9}^{(m)}=19^{m}-(4 m-64) 17^{m}+\left(8 m^{2}-252 m+1930\right) 15^{m}-
$$$$
\left(\frac{32}{3} m^{3}-496 m^{2}+\frac{22408}{3} m-36224\right) 13^{m}+
$$$$
\left(\frac{32}{3} m^{4}-\frac{1952}{3} m^{3}+\frac{43336}{3} m^{2}-\frac{412540}{3} m+469749\right) 11^{m}-
$$$$
\left(\frac{128}{15} m^{5}-640 m^{4}+\frac{55840}{3} m^{3}-260752 m^{2}+\frac{26162132}{15} m-4398784\right) 9^{m}+
$$$$
\left(\frac{256}{45} m^{6}-\frac{7552}{15} m^{5}+\frac{161792}{9} m^{4}-329280 m^{3}+\frac{145470664}{45} m^{2}-\right.
$$$$
\left.\frac{238528748}{15} \mathrm{~m}+29914578\right) 7^{\mathrm{m}}-
$$$$
\left(\frac{1024}{315} m^{7}-\frac{14848}{45} m^{6}+\frac{124928}{9} m^{5}-\frac{2803712}{9} m^{4}+\frac{179442208}{45} m^{3}-\right.
$$$$
\left.\frac{1290056152}{45} m^{2}+\frac{2193088532}{21} m-141218826\right) 5^{m}+
$$$$
\left(\frac{512}{315} m^{8} \frac{19456}{105} m^{7}+\frac{133888}{15} m^{6}-235520 m^{5}+\frac{55247168}{15} m^{4}-\right.
$$$$
\left.\frac{171886624}{5} m^{3}+\frac{57240313192}{315} m^{2}-\frac{9842673160}{21} m+379604650\right) 3^{m}-
$$$$
\left(\frac{2048}{2835} m^{9}-\frac{9728}{105} m^{8}+\frac{689152}{135} m^{7}-\frac{2361856}{15} m^{6}+\frac{400323968}{135} m^{5}-\right.
$$$$
\frac{522074336}{15} m^{4}+\frac{702603488128}{2835} m^{3}-\frac{102679699768}{105} m^{2}+\frac{75111559808}{45} m-
$$

264337010) 
TABLE XI. Some expressions for the coefficients $c_{j}^{(m)}$ (continued)

$$
\begin{aligned}
& 4^{20} c_{10}^{(m)}=21^{m}-(4 m-72) 19^{m}+\left(8 m^{2}-284 m+2462\right) 17^{m}- \\
& \left(\frac{32}{3} m^{3}-560 m^{2}+\frac{28696}{3} m-52944\right) 15^{m}+ \\
& \left(\frac{32}{3} m^{4}-736 m^{3}+\frac{55720}{3} m^{2}-202308 m+798079\right) 13^{m}- \\
& \left(\frac{128}{15} m^{5}-\frac{2176}{3} m^{4}+24032 m^{3}-\frac{1158704}{3} m^{2}+\frac{44900812}{15} m-\right. \\
& 8877288) 11^{\mathrm{m}}+ \\
& \left(\frac{256}{45} m^{6}-\frac{8576}{15} m^{5}+\frac{209792}{9} m^{4}-491200 m^{3}+\frac{252319864}{45} m^{2}-\right. \\
& \left.\frac{488845444}{15} m+74380386\right) 9^{m}- \\
& \left(\frac{1024}{315} m^{7}-\frac{5632}{15} m^{6}+\frac{813568}{45} m^{5}-\frac{1404416}{3} m^{4}+\frac{314705248}{45} m^{3}-\right. \\
& \left.\frac{895704688}{15} m^{2}+\frac{27937189928}{105} m-466277137\right) 7^{m}+ \\
& \left(\frac{512}{315} m^{8}-\frac{13312}{63} m^{7}+\frac{525568}{45} m^{6}-\frac{16048256}{45} m^{5}+\frac{294010624}{45} m^{4}-\right. \\
& \left.\frac{655243360}{9} m^{3}+\frac{149485794464}{315} m^{2}-\frac{169259066656}{105} m+2074575409\right) 5^{m}- \\
& \left(\frac{2048}{2835} m^{9}-\frac{32768}{315} m^{8}+\frac{1221632}{189} m^{7}-\frac{10179328}{45} m^{6}+\frac{658423424}{135} m^{5}-\right. \\
& \frac{2990989696}{45} m^{4}+\frac{319276747232}{567} m^{3}-\frac{875326272344}{315} m^{2}+ \\
& \left.\frac{2151552753452}{315} m-5348556339\right) 3^{m}+ \\
& \left(\frac{4096}{14175} m^{10}-\frac{131072}{2835} m^{9}+\frac{3056128}{945} m^{8}-\frac{122420224}{945} m^{7}+\frac{2205426688}{675} m^{6}-\right. \\
& \frac{7244229248}{135} m^{5}+\frac{1621995503776}{2835} m^{4}-\frac{10792995524608}{2835} m^{3}+ \\
& \left.\frac{22579172944784}{1575} m^{2}-\frac{1495484636944}{63} m+3674007443\right)
\end{aligned}
$$


TABLE XII. The polynomials $P_{p}(n ; j)$

$\begin{array}{rllll}j & P_{1}(n ; j) & P_{2}(n ; j) & P_{3}(n ; j) \\ 1 & 8 n+3 & & & \\ 2 & 8 n-4 & 64 n^{2}-64 n-34 & & \\ 3 & 8 n-12 & 64 n^{2}-176 n+60 & 512 n^{3}-2112 n^{2}+1504 n+942 \\ 4 & 8 n-20 & 64 n^{2}-304 n+292 & 512 n^{3}-3456 n^{2}+6112 n-1686 \\ 5 & 8 n-28 & 64 n^{2}-432 n+652 & 512 n^{3}-4992 n^{2}+14368 n-11088 \\ 6 & 8 n-36 & 64 n^{2}-560 n+1140 & 512 n^{3}-6528 n^{2}+25696 n-30096 \\ 7 & 8 n-44 & 64 n^{2}-688 n+1756 & 512 n^{3}-8064 n^{2}+40096 n-61776 \\ 8 & 8 n-52 & 64 n^{2}-816 n+2500 & 512 n^{3}-9600 n^{2}+57568 n-109200 \\ 9 & 8 n-60 & 64 n^{2}-944 n+3372 & 512 n^{3}-11136 n^{2}+78112 n-175440 \\ 10 & 8 n-68 & 64 n^{2}-1072 n+4372 & 512 n^{3}-12672 n^{2}+101728 n-263568\end{array}$

j $\quad P_{4}(n ; j)$

$44096 n^{4}-36864 n^{3}+98816 n^{2}-58944 n-41328$

$54096 n^{4}-51200 n^{3}+210176 n^{2}-299392 n+70968$

$64096 n^{4}-67584 n^{3}+385280 n^{2}-862656 n+580104$

$74096 n^{4}-83968 n^{3}+609536 n^{2}-1819328 n+1815288$

$84096 n^{4}-100352 n^{3}+882944 n^{2}-3267520 n+4212072$

$94096 n^{4}-116736 n^{3}+1205504 n^{2}-5305536 n+8304984$

$104096 n^{4}-133120 n^{3}+1577216 n^{2}-8031680 n+14726856$

j $\quad P_{5}(n ; j)$

$\begin{array}{rrr}5 & 32768 n^{5}-512000 n^{4}+2816000 n^{3}-6123520 n^{2}+3239552 n+2478720 \\ 6 & 32768 n^{5}-655360 n^{4}+4802560 n^{3}-15376640 n^{2}+19118592 n-4012560 \\ 7 & 32768 n^{5}-819200 n^{4}+7710720 n^{3}-33395200 n^{2}+63810112 n-38919000 \\ 8 & 32768 n^{5}-983040 n^{4}+11274240 n^{3}-61002240 n^{2}+152562112 n-135913440 \\ 9 & 32768 n^{5}-1146880 n^{4}+15493120 n^{3}-100160000 n^{2}+306230592 n-347689440 \\ 10 & 32768 n^{5}-1310720 n^{4}+20367360 n^{3}-152834560 n^{2}+549637312 n-749618400\end{array}$ 


\section{TABLE XII. The polynomials $P_{p}(n ; j)$ (continued)}

$P_{6}(n ; j)$

$6262144 n^{6}-6291456 n^{5}+57794560 n^{4}-248770560 n^{3}+474789376 n^{2}-$ $229256064 n$ - 188510400

$7262144 n^{6}-7667712 n^{5}+87654400 n^{4}-489000960 n^{3}+1338789376 n^{2}-$ $1511561088 n+285879600$

$8262144 n^{6}-9240576 n^{5}+129433600 n^{4}-909434880 n^{3}+3305084416 n^{2}-$ $5645881344 n+3196743840$

$9262144 n^{6}-10813440 n^{5}+179077120 n^{4}-1510809600 n^{3}+6761529856 n^{2}-$ $14911155840 n+12192150240$

$10262144 n^{6}-12386304 n^{5}+236584960 n^{4}-2324520960 n^{3}+12291083776 n^{2}-$ $32772475776 n+33789126240$

j $\quad P_{7}(n ; j)$

$72097152 n^{7}-71565312 n^{6}+983564288 n^{5}-6892462080 n^{4}+$ $25434472448 n^{3}-44282208768 n^{2}+19853396352 n+17363596320$

$82097152 n^{7}-84410368 n^{6}+1385431040 n^{5}-11841249280 n^{4}+$ $55522009088 n^{3}-136115348992 n^{2}+142793809920 n-24616141200$

$92097152 n^{7}-99090432 n^{6}+1930428416 n^{5}-19914424320 n^{4}+$ $115872985088 n^{3}-371756732928 n^{2}+583441743744 n-311292162720$

$102097152 n^{7}-113770496 n^{6}+2563506176 n^{5}-30906122240 n^{4}+$ $213465878528 n^{3}-833956680704 n^{2}+1671638425344 n-1277031616560$

$j \quad P_{8}(n ; j)$

$8 \quad 16777216 n^{8}-771751936 n^{7}+14797504512 n^{6}-152265293824 n^{5}+$ $898239627264 n^{4}-2974298865664 n^{3}+4830541058048 n^{2}-$ $2033894716416 n-1877990002560$

$9 \quad 16777216 n^{8}-889192448 n^{7}+19803406336 n^{6}-239681404928 n^{5}+$ $1698640297984 n^{4}-7040782794752 n^{3}+15879147393024 n^{2}-$ $15708098414592 n+2487754080000$

$10 \quad 16777216 n^{8}-1023410176 n^{7}+26438795264 n^{6}-375328866304 n^{5}+$ $3172917837824 n^{4}-16131399122944 n^{3}+47085624918016 n^{2}-$ $69097969483776 n+35099607070080$ 
TABLE XII. The polynomials $P_{p}(n ; j)$ (continued)

$j \quad P_{9}(n ; j)$

$9 \quad 134217728 n^{9}-8002732032 n^{8}+203893506048 n^{7}-2885777620992 n^{6}+$ $24649416572928 n^{5}-128707575349248 n^{4}+392752637673472 n^{3}-$ $603599318765568 n^{2}+240614820163584 n+233239937736960$

$10134217728 n^{9}-9059696640 n^{8}+262781534208 n^{7}-4268947931136 n^{6}+$ $42384027549696 n^{5}-262750997053440 n^{4}+991971647782912 n^{3}-$ $2094260870836224 n^{2}+1973487689726976 n-288763376630400$

j $\quad P_{10}(n ; j)$

$10 \quad 1073741824 n^{10}-80530636800 n^{9}+2629828608000 n^{8}-48911792209920 n^{7}+$ $568348190441472 n^{6}-4250313314795520 n^{5}+20235563712512000 n^{4}-$ $57890789535252480 n^{3}+85021138163810304 n^{2}-32282512864174080 n-$ 32707469473862400 